\title{
The Widely scalable Mobile Underwater Sonar Technology (WiMUST) H2020 project: first year status
}

\author{
Gianluca Antonelli*, Andrea Caffaz ${ }^{\dagger}$, Giuseppe Casalino*, Ivan Bielic de Jong ${ }^{\ddagger}$, Daniela De Palma*, \\ Henrique Duarte ${ }^{\S}$, Jonathan Grimsdale ${ }^{\Uparrow}$, Giovanni Indiveri*, Sergio Jesus ${ }^{\|}$, Konstantin Kebkal ${ }^{* *}$, \\ Antonio Pascoal ${ }^{\dagger \dagger}$, Daniel Polani ${ }^{\ddagger \dagger}$ and Lorenzo Pollini* \\ ${ }^{*}$ ISME, Italy \\ ${ }^{\dagger}$ Graal Tech, Italy

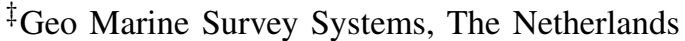 \\ $\S_{\text {Geosurveys Consultores, Portugal }}$ \\ ${ }^{\ddagger}$ Geo Marine Survey Systems, The Netherlands \\ CGG, France \\ $\|_{\text {CINTAL, Portugal }}$ \\ **EvoLogics, Germany \\ ${ }^{\dagger \dagger}$ IST, Portugal \\ ${ }^{\dagger \dagger}$ Univ. of Hertfordshire, United Kingdom
}

\begin{abstract}
The Widely scalable Mobile Underwater Sonar Technology (WiMUST) project is an H2020 Research and Innovation Action funded by the European Commission. The project aims at developing a system of cooperative Autonomous Underwater Vehicles (AUVs) for geotechnical surveying and geophysical exploration. The paper reports about the first year activities and it gives an overview of the main objectives and methods.
\end{abstract}

\section{INTRODUCTION}

WiMUST (Widely scalable Mobile Underwater Sonar Technology) is an ongoing project funded by the European Community within the H2020 framework (Work Programme 2014 - 2015, LEIT- ICT, 5. Leadership in enabling and industrial technologies - Information and Communication Technologies). The ultimate goal of the project is to design and test a system of cooperating Autonomous Underwater Vehicles (AUVs) able to perform innovative geotechnical surveying operations. In particular, the WiMUST system will be composed by a small fleet of AUVs carrying hydrophones to acquire sub-bottom profiling acoustic data. Contrary to the classical technology based on ship towed streamers, the WiMUST solution will allow to change the geometry of the acoustic antenna: something that has not been achieved in practice and holds potential to significantly improve ocean surveying. This paper aims at giving a brief overview of the project [1] [2]. In particular this paper is an updated and extended version of [3]. Section II addresses the main concepts and approaches. Section III focuses on the distributed sensor array issues to be faced in the project. Section IV describes the AUV cooperative control activities while Section V gives an overview of the mission planning issues to be faced in the project. Section VI addresses the communication activities within the project and Section VII briefly accounts for the necessary system integration and experimentation activities. Finally Section VIII reports concluding remarks.

\section{CONCEPT AND APPROACH}

The WiMUST project [3] aims at conceiving, designing, and engineering an intelligent, manageable, distributed and reconfigurable underwater acoustic array that could significantly improve the efficacy of the methodologies used to perform geophysical and geotechnical acoustic surveys at sea. The use of the WiMUST system will be beneficial in a vast number of applications in the fields of civil engineering and oil \& gas industry, where seabed mapping, seafloor characterization, and seismic exploration are fundamental operations. The novel key feature of the WiMUST system consists in the use of a team of cooperative autonomous marine robots, acting as intelligent sensing and communicating nodes of a reconfigurable moving acoustic network. The vehicles are equipped with hydrophone streamers of small aperture, such that the overall system behaves as a distributed acoustic array capable of acquiring acoustic data obtained by illuminating the seabed and the ocean sub-bottom with strong acoustic waves sent by one (or more) acoustic source installed on-board a support ship / boat.

By actively controlling the geometry of the robot formation, it becomes possible to change the shape of the acoustic array, according to the needs of the considered application. The resulting operational flexibility holds potential advantages, as it allows improving the seabed and sub-bottom resolution and obtaining sidelobe rejection at almost any frequency and for any plane. The availability of the proposed system, other than improving the quality of the acquired data, will also greatly facilitate the operations at sea, thanks to the lack 
of physical ties between a surface ship and the acquisition equipment. As a preliminary step for the research work of the project, the activities have started with an analysis of the reference scenario which needs to be characterized in terms of desired functionalities and expected behavior of the WiMUST system. In particular, the reference scenarios will cover both the 2D and 3D active geoacoustic exploration and geotechnical seafloor characterization scenarios. As a result, specifications for all the composing subsystems (mission planning, communication, distributed sensing, navigation, coordination) will be drawn out, together with a preliminary indication of the expected corresponding hardware resources (sensors, communication devices, infrastructures).

\section{Distributed SENSOR ARRAY}

One of the most ground breaking proposals in WiMUST is that the AUV-based acoustic sensing array is physically disconnected from the source system which allows for a new freedom in sensing array geometry. If we abstract from the physical constraints relative to vehicle navigation, speed, positioning and other limitations, the WiMUST sensing system may be viewed as a distributed sensor array (DSA) free to adopt any geometry. Moreover, that geometry may change along time, upon request. So, in terms of data processing, the fundamental question to be tackled is whether the seismic data acquisition and processing protocol established for ship towed streamers is also suited for the mobile AUV-based acoustic sensing system? And, if not, in which situation and how should it be modified. Determining the geophysical properties of the ocean bottom is an old and non trivial problem with scientific as well as engineering implications. Even if electromagnetic approaches exist [4] the large majority of the survey methods are based on the analysis of bottom acoustic returns complemented with in situ observations and historical information. The problem is complicated by the fact that sound velocity strongly varies in consolidated sediments, which makes it difficult to determine at the same time bottom layering (i.e., layer thickness), based on the time-of-flight of acoustic arrivals, and compressional velocities on each layer where those arrivals propagate. This makes the problem nonlinear. There are two main classes of methods to address this problem: one is based on the matching of the observed data with the acoustic full-field calculated for a physical model of the bottom, including layering and layer physical properties. This is the so-called matched-field inversion (MFI) class of methods. The other is based on transforming the non-linear problem into two linear steps: in the first step the time-offlight of identified bottom returns are matched assuming a given velocity model (based on a priori knowledge); in the second step, arrival times are successively matched with those obtained from the data and, as later arrivals are considered, the grazing angle changes and the focal point moves deeper into the bottom, which requires successive adjustment of the velocity model until a good match is obtained. This is the approach used in classic time-of-arrival (ToA) seismic surveying [5]. For various reasons MFI is more often used with vertical arrays and for high-resolution of surficial sediments in shallow water, while ToA is used with horizontal (towed) arrays, both in shallow and deep water, for low resolution bottom imaging of large high intensity reflectors deeply buried into the bottom. The latter is the typical problem faced in oil and gas exploration where the objective is to detect a salt crust sometimes one or more km below the ocean bottom. In order to motivate the approach taken in this work we start by considering a highly conceptual view where we attempt to "explain" the data $\mathbf{d}$ with a model $\mathbf{m}\left(\mathbf{p}_{0}\right)$ that depends on parameter vector $\mathbf{p}_{0}$. The sought performance criteria is obviously dependent on how close, in the least squares sense, parameter vector estimate $\mathrm{p}$ is of true vector $\mathbf{p}_{0}$. Therefore the problem may be stated as follows

$$
\min \left\|\mathbf{p}-\mathbf{p}_{0}\right\|_{2} \text { s.t. } \mathbf{d}=\mathbf{m}(\mathbf{p})
$$

which can not be solved for $\mathbf{p}$ since $\mathbf{p}_{0}$ is unknown. One way to circumvent this is to separate $\mathbf{p}$ into two parts: the layering $\mathbf{x}$ and the remaining parameters $\boldsymbol{\theta}$, namely the sound velocities in the considered layers. With this assumption, model $\mathbf{m}$ may be written under a linear form as $\mathbf{m}=\boldsymbol{A}(\boldsymbol{\theta}) \mathbf{x}$. Although unknown, the number of effective layers in $\mathbf{x}$ is much smaller than the actual measurements in $\mathbf{m}$ (or $\mathbf{d}$ ), than model $\mathbf{m}=$ $\boldsymbol{A}(\boldsymbol{\theta}) \mathbf{x}$ may be viewed as a sparse system of equations and the problem in (1) rewritten as

$$
\min \|\mathbf{x}\|_{1} \text { s.t. } \mathbf{d}=\boldsymbol{A}(\boldsymbol{\theta}) \mathbf{x},
$$

where now the minimization is over the non zero components of sparse vector $\mathbf{x}$. If there is noise in the observations $\mathbf{d}$, now taken as $\mathbf{y}=\mathbf{d}+\mathbf{n}$, where $\mathbf{n}$ is the noise, (2) reduces to

$$
\min \|\mathbf{x}\|_{1} \text { s.t. }\|\mathbf{y}-\boldsymbol{A}(\boldsymbol{\theta}) \mathbf{x}\|_{2}<\boldsymbol{\delta}
$$

For $\boldsymbol{\theta}$ known (or estimated) and $\boldsymbol{A}$ full rank, even if the number of observations is much smaller than the dimension of vector $\mathbf{x}$, (2) is a problem that can be solved by linear programming using an efficient basis-pursuit algorithm subject to fitting constraints. The solution depends on the structure of matrix $\boldsymbol{A}$ that has less rows than columns and therefore should guarantee, with a high probability, that the non-zero entries of $\mathbf{x}$ are observable in $\mathbf{d}$ ( or $\mathbf{y}$ ). This is the problem faced in compressed sensing where it was shown that the condition is that $\boldsymbol{A}$ should have a low coherence [6]. Low coherence means that $\boldsymbol{A}$ should be as "flat" as possible. The practical problem in our case is that matrix $\boldsymbol{A}$ is formed by the acoustic propagation model that connects the source, the bottom layers and the receivers, i.e., the Green function of the media (constrained in parameter $\boldsymbol{\theta}$ ) and which can not be changed. In practice the coherence of matrix $\boldsymbol{A}$ may be lowered by selecting observations (the rows of $\boldsymbol{A}$ ) at random positions [7] [8]. Low coherence means high diversity which is simply equivalent to placing the sensors of our DSA at positions where the bottom received acoustic field is the most diverse or where it shows the lowest coherence. In other words our conviction is that the criteria for determining the optimal DSA geometry should follow the line of low coherence or high diversity within, 
of course, the operational and practical constraints of the WiMUST AUV-based platform. Figure 1 illustrates the results

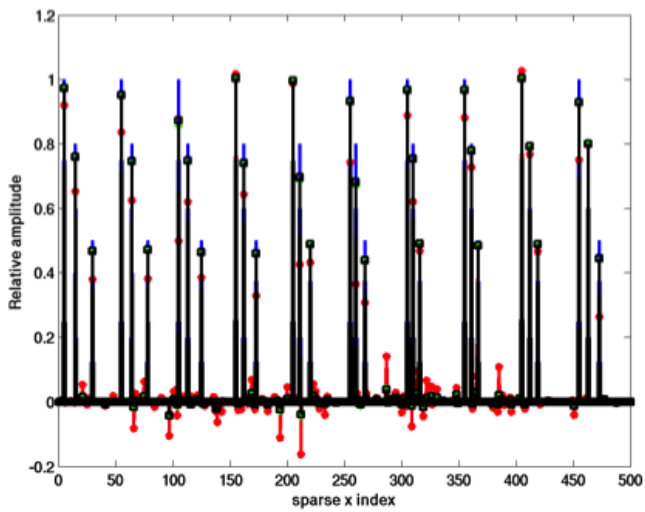

(a)

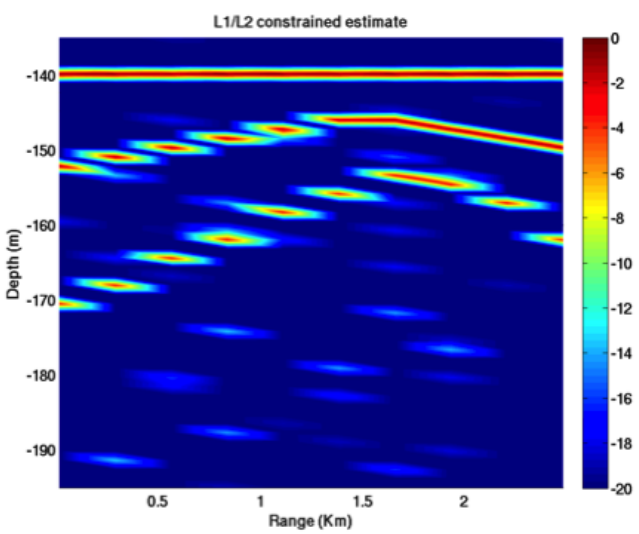

(b)

Fig. 1. Inversion results on synthetic example with 30 bottom scatterers using the YALL [9] package for the $l_{1}$ norm (red), for the $l_{1}$ norm $l_{2}$ unconstrained (green) and for the $l_{1}$ norm $l_{2}$ constrained, corresponding to 3 , (black) and true values (blue) in (a) and bottom reconstruction with the $l_{1}$ norm $l_{2}$ constrained (b).

obtained in a 2D example where a synthetic environment composed of a $140 \mathrm{~m}$ deep water layer, two 10 and $20 \mathrm{~m}$ thick sediment layers over a sub-bottom half space with variable compressional speed from 1550 up to $2500 \mathrm{~m} / \mathrm{s}$, excited by a $500 \mathrm{~Hz}$ sound source at $5 \mathrm{~m}$ depth. The receiver is a linear 100 sensor array with $2500 \mathrm{~m}$ aperture also at $5 \mathrm{~m}$ depth. A bottom grid of $50 \times 10$ samples was defined for a bottom coverage of $60 \mathrm{~m}$ depth $\mathrm{x} 2500 \mathrm{~m}$ range. A number of up to $\mathrm{K}=30$ bottom scatters where simulated in a peak shaped deeper sediment layers. The acoustic propagation model OASES [10] was used to generate the array received field where an SNR of $10 \mathrm{~dB}$ was used. The 100 receivers where randomly distributed and a coherence of 1.3 was obtained for the example shown (note that the used normalization implies that the coherence varies between 1 and square root of the number of search sample, in this case 22). Three optimization algorithms from the YALL package [9] were tested and the results are shown in figure 1 for the $l_{1}$ norm (see (2)) showing a large number of spurious peaks (red), for the $l_{1}-l_{2}$ norm unconstrained where still some low amplitude estimates at wrong depths can be seen (green) and for the $l_{1}-l_{2}$ norm constrained, corresponding to (3), with the lowest mean square error (black) when compared to the true values (blue) (figure 1a). At each range the three different amplitudes denote the reflector strength decrease with depth into the bottom. The results obtained with the $l_{1}-l_{2}$ norm constrained where used to reconstruct the $2 \mathrm{D}$ bottom estimate along range and depth giving a visual perception of the bottom layering structure (figure 1b). The above methodology requires further testing for variable sensing array geometries, broadband signals and robustness testing to various environmental parameter $\boldsymbol{\theta}$ mismatch. A simulator for performance prediction in realistic data will normally follow with application to real data when available.

\section{CoOperative CONTROL}

Cooperative navigation, guidance, and control of the AUV robotic vehicle team is one of the pillars of WiMUST system. The WiMUST vehicles will need accurate relative navigation and control capabilities, ensuring inter-vehicle collision avoidance and yielding a virtual structure to accurately position each hydrophone streamer, thereby shaping the formation of the resulting sonar receiving array. Acquiring seismic data with well defined characteristics requires the acoustic source and each of the acoustic receivers to maintain a specific geometric formation. In the scope of WiMUST, this translates into a clear control task: steer each vehicle so that all vehicles, the streamers being towed, and the acoustic source (usually a sparker) maintain a specified formation geometry. We make the simplifying, yet realistic assumption that the trajectory followed by the source (usually towed from a support vessel) is known with an adequate degree of accuracy, usually obtained from a DGPS or RTK GPS installed on the source. At this point in the project, two types of candidate solutions arise for this control problem, each with its advantages and disadvantages. We now give a brief overview of each of these solutions, as they currently stand, and propose modifications and application scenarios in the WiMUST setting.

\section{A. Cooperative path following}

The Cooperative Path Following (CPF) control algorithm assumes that each vehicle has full knowledge of the path to be followed in a close future, with no associated timing law, and that the paths of all vehicles are parametrized with an along-path coordinate $\gamma$. The goal of the control algorithm is that all vehicles follow the path in a synchronized fashion, i.e. the vehicles are at "corresponding" points (points with the same $\gamma$ coordinate) on their own paths at each instant of time. Therefore, the parametrization of the paths must take into account the desired formation geometry. The absence of an associated timing law implies that the algorithm does not impose where each individual vehicle should be at each instant in time - just that all vehicles should be at corresponding points on their paths. However, in cooperative path following there is room to specify desired speeds as functions of the parameter 
$\gamma$ and not of time explicitly; this is called speed assignment. The main idea behind the algorithm, as described formally in [11], where a proof of convergence is given, is that each vehicle $i$ keeps track of its own "virtual target" (a point on the path, with an associated along-path coordinate ?i). While each vehicle attempts to steer towards its target, the targets themselves are adjusted (slowly) based on the targets of the other vehicles in the formation, so that:

i) they converge to the same value of $\gamma$ on their respective paths;

ii) their speeds $d \gamma / d t$ along the respective paths converge to desired values, as determined by the specific mission being executed.

In the scope of WiMUST, one of the elements in the

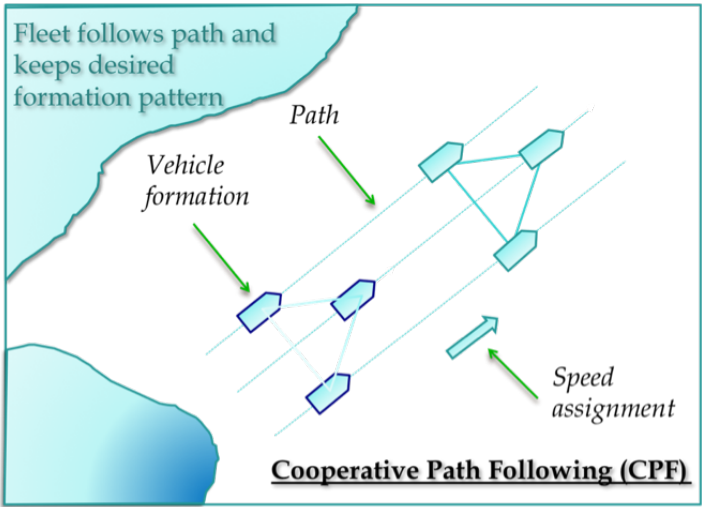

Fig. 2. A schematic drawing of a formation running the CPF algorithm and maintaining a triangular geometry

formation to be maintained is the acoustic source. As the source is towed by a human-piloted vessel, this element should be considered not-cooperative in the sense that its "virtual target" cannot be adjusted in real time in response to the motion of the vehicles. As such, in order to use a CPF-like algorithm, a modification must be introduced. A possible idea is to consider that the formation includes all the vehicles, but not the acoustic source, and replace requirement (ii) above with a prescribed value on position (along-path coordinate) as a function of time, rendering the algorithm closer to a tracking approach, as described later. This will be subject of further investigation, since it is not known whether introducing this requirement compromises the desirable robustness and stability characteristics of the CPF algorithm.

The main advantage of this algorithm lies in its robustness to failure of a single element. The particularly simple case of a formation consisting of several vehicles moving along a common straight line is an example of especial importance. In this case, some kind of inter-vehicle cooperation would ideally exist so that if one of the vehicles in front of the formation fails and stops, the remaining vehicles behind it also eventually slow down and stop, regardless of the motion of the support vessel towing the source. In contrast, the main disadvantages are that:
- a-priori information about the paths to be followed is needed;

- the algorithm requires the vehicles to exchange information (their along-path coordinates $\gamma_{i}$ ) at a considerably high rate, so that the $\gamma$ coordinates can be updated regularly to achieve synchronization.

Moreover, communication strategies involving frequent information exchange between all vehicles cannot be used in an underwater environment without compromising scalability the frequency of this information exchange would decrease linearly as the number of vehicles increases. Therefore, a solution purely based on CPF-like algorithms can be used only when all the vehicles move at the surface, so that this information exchange can occur via WiFi instead of acoustic networks.

\section{B. Target/trajectory tracking}

The target/trajectory tracking (TT) family of algorithms, described and analyzed formally in [12] and then applied to specific scenarios in [13] [14], resorts to a fundamentally different approach. The goal is for a single vehicle to follow a trajectory with an associated timing law, i.e. enforcing where the vehicle should be at specific times. The idea behind this is that, again, a vehicle tries to steer towards its "virtual target", but this target moves solely based on a specific timing law. This implies that for each time instant $t$ there is a prescribed desired position for the vehicle, $\mathbf{p}_{d}(t)$, and optionally other references such as desired heading and depth. The algorithm can then be naturally extended [14] to achieve geometric formations in multiple-vehicle missions by choosing the trajectory (including the spatial path and its associated timing law) for each vehicle appropriately for the desired geometry. Then, when all vehicles eventually converge to their own targets, the formation moves in a synchronized fashion and this desired geometry is maintained.

In practice, a major drawback of this algorithm when compared to path-following is its lack of robustness, in the sense that if some unplanned event happens (e.g. ones of the vehicles cannot follow its target) the desired geometry can no longer be maintained. However, some characteristics of this algorithm seem to fit the particular WiMUST case, namely:

- no a-priori knowledge of the trajectory is needed - it suffices that at each time instant each vehicle can compute the position and velocity vector of its virtual target, based on information about the motion of the acoustic source, for example;

- the "time-dependent" nature of the algorithm suits the control objective: the desired position of each vehicle, at any specific time instant, depends on the position of the acoustic source at that time. In other words, each vehicle should be at the right place at the right time (there is no point in following the same path as the acoustic source if not with the correct timing);

- no high-frequency information exchange between the vehicles is needed, as each vehicle can compute the position and velocity its virtual target based solely on information 
about its own position and velocity and those of the acoustic source. This allows TT-like algorithms to be applied even in an underwater setting where the vehicles can communicate only via the acoustic network and, as stated before, high- frequency information exchange between all vehicles is not possible for scalability reasons.

\section{Hybrid approaches}

Having stated the main characteristics of these two types of geometric formation control algorithms, it seems that some hybrid approach would be desirable in the context of WiMUST. Ideally, the algorithm should meet the high-level control objective - to keep the vehicles in a desired geometry with respect to the acoustic source - while borrowing some of the robustness characteristics of the CPF and, for the underwater case, avoiding the need for frequent inter-vehicle communication. The application of possible hybrid approaches will certainly be a topic of further study within the project. An example of an alternative to be investigated is the case where the future trajectory of the acoustic source is predicted on a time-window, based on past and present position and velocity vectors, and a CPF-like algorithm is ran using this predicted path.

\section{Single range navigation and guidance}

During the initial phase of each mission, when the vehicles move towards a given waypoint in order to reach the desired formation, a single range aided navigation could be used to improve the localization performance. To this effect, a novel strategy has been proposed in [15] that addresses the problem of driving an underwater vehicle to a desired position while optimizing the performance of a single-range based localization system. Indeed, the performance of single-range based localization techniques is strongly dependent on the vehicle?s motion that needs to be sufficiently rich to yield a good measure of observability. A prioritized task oriented technique to combine a point-to-point guidance task with an observability optimization task can be used. In particular, the minimum singular value of the Fisher Information Matrix is adopted as the observability metric. Task prioritization is achieved through a null-space-based projection technique that is also combined with a saturation management algorithm to cope with velocity command saturations. The stability of the resulting control law is analyzed following a Lyapunovbased approach. Robustness against uncertainty in the initial position of the vehicle can be also handled resorting to a procedure that maximizes the minimum (worst-case) observability metric. Numerical simulations reported in [15] confirm the effectiveness of the proposed strategy.

\section{MISSION PLANNING}

The execution of WiMUST missions will require the availability of flexible mission planning algorithms for the deployed robotic units. An innovative approach towards mission planning is developed which extends classical algorithms for mission planning by novel intelligent methodologies. The proposed approach is based on the information-theoretic quantity empowerment [16] [17], which is associated to the state of a robot and can be exploited to generate preferred behaviors without having to resort to specialized cost functions, which usually vary from task to task and have to be hand-designed. Technically speaking, empowerment is defined as the Shannon channel capacity between the robot controls and its states in subsequent time-steps, therefore it is measured in bits. Its computation is based on adaptive evaluation of the acting-sensing interaction of the robot group with its environment. Within the WiMUST project empowerment is used for the first time in the context of marine robotics. In this context its definition makes explicit use of marine systems dynamic modeling, taking into account single vehicle hydrodynamic effects (added masses, viscous frictions, restoring torques and forces, currents, etc.). Empowerment was already proven to identify desirable states for agents, in term of intrinsic survivability and robustness drives, in a wide spectrum of scenarios [18]. In fact it can be considered a information-theoretic generalization of the concept of mobility (in principle, a quantitative metrics of the distinct controllably accessible states). Essentially it measures to what extent an agent can influence the environment by its controls: it is zero if, regardless what the robot does, the outcome of the controls will be the same; it is maximal if every control will have a distinct outcome. In WiMUST we investigate how empowerment can be used as a vehicle-intrinsic metric to evaluate autonomous underwater vehicles' desirable trajectories in terms of mobility and survivability. Hence, while performing team formation control, the overall WiMUST mission planner may benefit by the possibility of monitoring online the empowerment of the state of each vehicle. Since empowerment is zero in situations where the vehicles have no mobility at all, one rationale for its use is to detect the robots being in or approaching undesirable conditions as being stuck or crashed. In addition, empowerment can play the role of a purely intrinsic utility function that serves to enhance the control of the vehicles during a mission; we utilize its property as a powerful heuristic to improve situation-adapted and flexible reaction in safe-critical scenarios. This can be particularly relevant in emergency situations when the vehicle has no access to an explicitly specified external cost function to minimize (e.g. because it lost communication with the base ship). To this end we develop an efficient empowerment-based controller for vehicles that will eventually follow their own empowerment gradient (i.e. they will choose controls which lead to successor states with the highest empowerment). The key innovation of the empowerment-based approach is to move away from inflexible hand-designed algorithms to a flexible approach that has, in many example scenarios, demonstrated the ability to incorporate situation-specific response capability. To this end, we intend to show that the utilization of empowerment-based mission control can be used to make the vehicles improve their own survivability autonomously, without having to explicitly specify this requirement within the mission definition. We hypothesize that this approach will 
endow the WiMUST mission planner the "graceful degradation" property, so as to prevent the basic functionalities of the vehicles to break down entirely when subcomponents of the WiMUST control system may be inoperative.

\section{COMMUNiCATIONS}

The main objectives of the communication related research within WiMUST are the development of algorithms and procedures for accurate distance measurements (up to the centimeter scale) between AUVs moving in formation, reconstruction of their relative positions in $3 \mathrm{D}$ space, and precise synchronization of their local clocks. These are required to assign temporal and spatial tags to acquired data that are consistent across the formation of vehicles, and to support some modalities of navigation that require explicit relative positions to control the shape of the formation of vehicles. Moreover, it will be necessary to achieve an estimation of practical data throughput boundaries for different AUV formations. Based on such estimates, specific work will address the development of communication algorithms and procedures to support the different operative and environmental conditions. The work in this area will be organized along two major lines: long range and short range communication issues. As for the short range communication requirements, high bit rate communication of AUVs at short range is a pre-requisite for the design of cooperative teams of underwater vehicles for the operations envisioned in the WiMUST applications. Typical bit rates by conventional acoustic modems are rather low usually reaching, in practice, effective bit rates on the order of several hundred bits per second having nominal bit rates of several kilobits per second. Recent modem developments made by EvoLogics provided a significant increase of practically achievable bit rates. The objective is to focus on the evaluation of practically achievable bit rates within the necessary team geometries of practical interest, which will enable successful implementation of the navigation and motion control tasks within the project. Accounting for specific AUV formations and typical environmental conditions, the achievable bit rates will be firstly obtained by modeling and simulation and then compared with the results of practical experiments. In this manner, the basic estimates of currently achievable bit rates can be accounted for the needs of the project. To enable a reliable communication necessary to adequately control the AUVs formation, a robust data transmission with the use of advanced networking protocols will be implemented and tested. Moreover, specific work will aim at developing a decentralized clock synchronization algorithm based on the data exchange using short range link. The algorithm will allow to keep mutual clock drifts much smaller than for traditional computer clocks. The overall short range communication system will allow to extend the existing point-to-point algorithms of distance measurement in the WiMUST team of AUVs. The challenge here is to provide sufficiently accurate distance measurement in the distributed network of moving vehicles. While improving the data rates and reducing the packet overhead in point-to-point communications is of paramount importance, an equally relevant issue is to design decentralized localization and clock synchronization algorithms for efficient use of the transmission medium (see, e.g., [19], [20], [21]). This need arises due to the potentially large number of vehicles that must share the acoustic channel, which may quickly lead to a saturation of resources in the network as a whole even if individual packets are transmitted as fast as the channel allows. It is therefore necessary to develop streamlined versions of distributed estimation algorithms that take advantage of the broadcast nature of the medium to reduce the amount of data that each node must share with others to preserve valuable channel resources (in addition to all optimizations that may be undertaken at the level of networking protocols), without unduly sacrificing estimation accuracy. While active research is being performed within the project relative to the clock synchronization issue, it is planned that the WiMUST AUVs will be equipped with atomic clocks enabling the WiMUST system to properly function for the duration of a typical mission in spite of possible poor clock synchronization algorithm performance. As for the long range communication requirements of the overall system, the exploitation of advantages given by sweep-spread communication technology of EvoLogics opens the way to practically achievable bit rates of about a several to tens kilobit/s in long range. While the range of data transmission and the capacity of the underwater acoustic channel may change vigorously in consequence of changes in environmental conditions, an efficient implementation of an acoustic communication network will strongly depend on the capability of the modem to automatically adjust its bit rate to the actual channel conditions. Since parallel / asynchronous transmission of many differently prioritized data streams from one or many data sources represents one of standard features of EvoLogics modems, the communication bit rate can be adapted to the WiMUST operating conditions.

\section{INTEGRATION AND EXPERIMENTATION}

As described in [3], specific activities for system integration are planned within the project: indeed, all the subsystems and functionalities developed within the project will need to be integrated to form the final WiMUST system. Moreover, the overall system will be validated through experimental tests at sea. The vehicles constituting the WiMUST system will need to perform cooperative guidance, navigation - localization and control by implementing the solutions and methods derived in the action. In the final experiment, the WiMUST vehicles will need to exhibit a sufficient degree of autonomy and intelligence in controlling the required formation while concurrently performing operational tasks related to the level of individual power supply, intra-vehicle distance, and quality of service of vehicle-to-vehicle communication. The final experimental tests will thus serve as a validation for all project goals. Such tests are planned to be executed either in the Setubal area (Portugal) or close to the Elba island (in Italy) (figures 3 - 4).

With reference to the system integration activities, these will initially deal with mechatronic integration of the sensing payloads and the communication devices on board the 


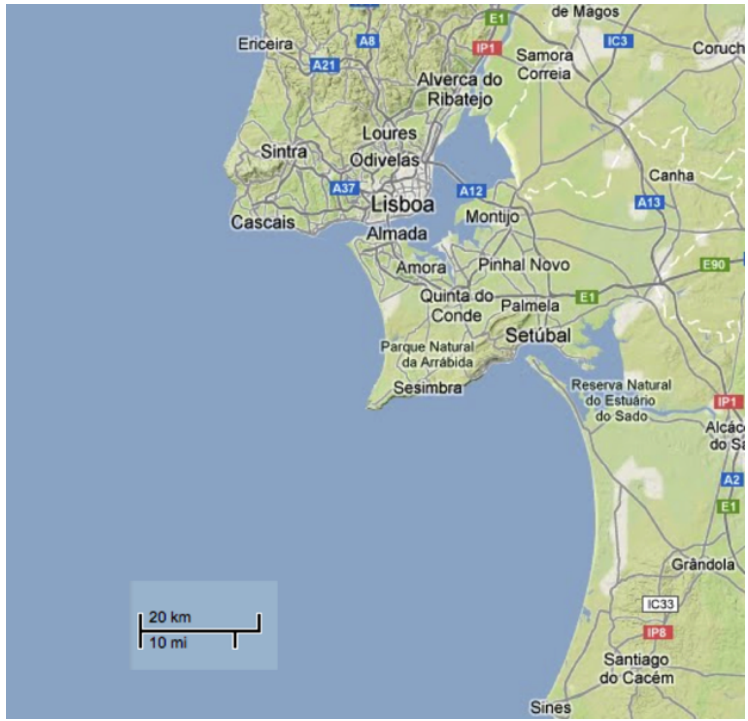

Fig. 3. Setubal (Portugal) area: one of the candidate sites for the final WiMUST tests.

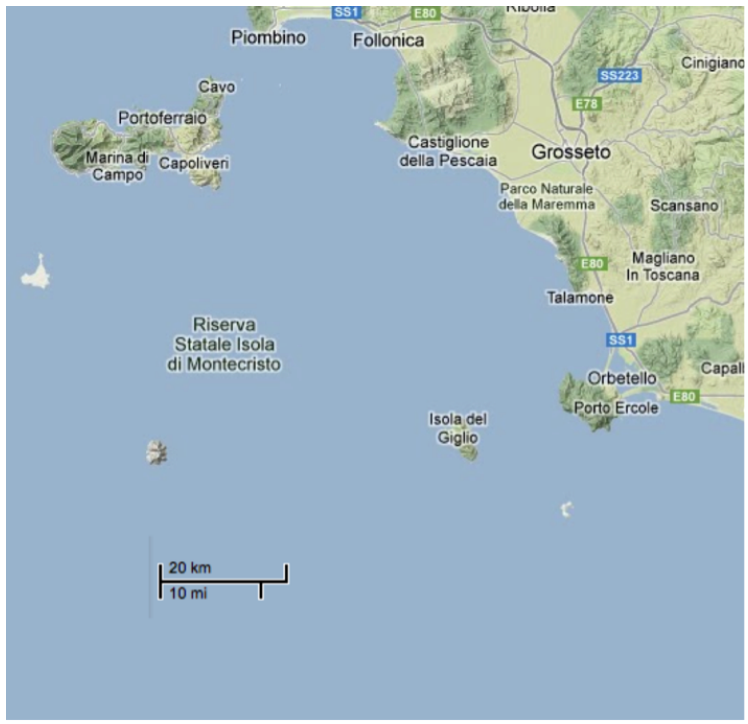

Fig. 4. Elba island (Italy) area: one of the candidate sites for the final WiMUST tests.

AUVs. Successively, the software modules concerning group navigation and coordination will be finalized and inserted in the final AUVs' software architecture together with the distributed sonar algorithms and the communication protocols and strategies. Once the basic functionalities will be validated in lab and through the "wet" engineering experiments, sea trials on the overall WiMUST system will be executed. Data gathered during experiments will be finally analyzed for obtaining indications on the real performance of the WiMUST system.

Preliminary tests of the MEDUSA class AUVs from the WiMSUT partner Instituto Superior Técnico (IST, Lisbon, Portugal) towing a streamer (from partner GEOMARINE, The Netherlands) have been performed in late 2015 / early
2016. The streamers used for these tests are actually "dummy" streamers, namely without any internal cabling, hydrophones, and pre-amplifiers. Other than this, they use exactly the same materials and have the same geometry that is envisioned for the WiMUST project. Two dummy streamers were manufactured specifically for this purpose by GEOMARINE and kindly made available to IST. The mass of each streamer is 19.0 $\mathrm{kg}$. In the preliminary test experiments in Lisbon (Figure 5), two missions were carried out in which the MEDUSA vehicle, towing the streamer, followed circular trajectories of radii 12 $\mathrm{m}$ and $8 \mathrm{~m}$ respectively. A small, human- piloted support boat followed the tip of the streamer, and a GPS antenna fixed to a bar was manually kept on top of it. This produced GPS position data for both the MEDUSA vehicle and the tip of the streamer. For each of the circular missions, the streamer tip was indeed found to follow a circumference of a smaller radius than that of the MEDUSA, and a radii difference between the two was computed for each of the missions $(12 \mathrm{~m}$ and $8 \mathrm{~m}$ for the MEDUSA). The acquired data will be instrumental to identify a dynamic model of the AUV - streamer system that is of great importance for navigation, guidance and control of the overall system.

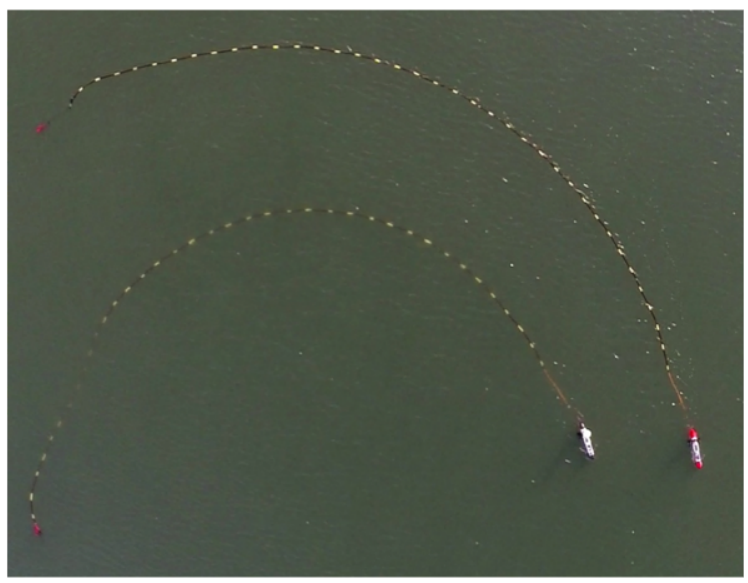

Fig. 5. Aerial view of two MEDUSA vehicles (IST) towing two streamer in a curve.

\section{CONCLUSION}

The vision underlying the Widely scalable Mobile Underwater Sonar Technology (WiMUST) project is that of developing advanced cooperative and networked control / navigation systems to enable a large number (tens) of marine robots (both on the surface and submerged) to interact by sharing information as a coordinated team (not only in pairs). The WiMUST system may be envisioned as an adaptive variable geometry acoustic array. This paper has provided an overview of the WiMUST objectives and its methods.

\section{Acknowledgments}

This work has received funding from the European Union's Horizon 2020 research and innovation programme under grant agreement No. 645141 (WiMUST project). 


\section{REFERENCES}

[1] H. Al-Khatib, G. Antonelli, A. Caffaz, A. Caiti, G. Casalino, I. B. de Jong, H. Duarte, G. Indiveri, S. Jesus, K. Kebkal, A. Pascoal, and D. Polani, "Navigation, guidance and control of underwater vehicles within the widely scalable mobile underwater sonar technology project: an overview," in 4th \{IFAC\} Workshop onNavigation, Guidance and Controlof Underwater Vehicles $\{N G C U V\}$ 2015 Dedicated to the memory of Professor Geoff Roberts, vol. 48, no. 2, 2015, pp. 189 - 193. [Online]. Available: http://www.sciencedirect.com/science/article/pii/S2405896315002700

[2] G. Indiveri and J. a. Gomes, "Geophysical surveying with marine networked mobile robotic systems: The WiMUST project," in WUWNET '14 Proceedings of the International Conference on Underwater Networks \& Systems, Rome, Italy, November 12-14 2014 [Online]. Available: http://dx.doi.org/10.1145/2671490.2677084

[3] H. Al-Khatib, G. Antonelli, A. Caffaz, A. Caiti, G. Casalino, I. B. de Jong, H. Duarte, G. Indiveri, S. Jesus, K. Kebkal, A. Pascoal, and D. Polani, "The widely scalable mobile underwater sonar technology (WiMUST) project: an overview," in Proceedings of MTS/IEEE Oceans '15, Genova, Italy, May 18-21 2015, pp. 1-5. [Online]. Available: http://dx.doi.org/10.1109/OCEANS-Genova.2015.7271688

[4] A. Ziolkowski and D. Wright, "The potential of the controlled source electromagnetic method: A powerful tool for hydrocarbon exploration, appraisal, and reservoir characterization," IEEE Signal Processing Magazine, vol. 29, no. 4, pp. 36-52, July 2012. [Online]. Available: http://dx.doi.org/10.1109/MSP.2012.2192529

[5] Ö. Yilmaz, Seismic Data Processing. SEG, Tulsa, 1987.

[6] E. Candès and J. Romberg, "Sparsity and incoherence in compressive sampling," Inverse Problems, vol. 23, 2007. [Online]. Available: http://dx.doi.org/10.1088/0266-5611/23/3/008

[7] A. Fannjiang, P. Yan, and T. Strohmer, "Compressed Remote Sensing of Sparse Objects," arXiv.org, 2009. [Online]. Available: http://arxiv.org/abs/0904.3994

[8] S. Jesus, "Distributed sensor array for bottom inversion," in IEEE/OES China Ocean Acoustics'2016, Harbin (China), 9-11 January 2016.

[9] J. Y. Y. Zhang and W. Yin, YALL package. Rice University, 2010.

[10] H. Schmidt, OASES 3.1 - User guide and reference manual. MIT, March 2011

[11] R. Ghabcheloo, A. P. Aguiar, A. Pascoal, C. Silvestre, I. Kaminer, and J. Hespanha, "Coordinated path-following in the presence of communication losses and time delays," SIAM - Journal on Control and Optimization, vol. 48, no. 1, pp. 234-265, 2009. [Online]. Available: http://dx.doi.org/10.1137/060678993

[12] P. C. Abreu and A. M. Pascoal, "Formation control in the scope of the morph project. part i: Theoretical foundations," in 4th IFAC Workshop onNavigation, Guidance and Controlof Underwater VehiclesNGCUV 2015, vol. 48, no. 2. Girona, Spain: IFACPapersOnLine, 28-30 April 2015 2015, pp. 244-249. [Online]. Available: http://dx.doi.org/10.1016/j.ifacol.2015.06.040

[13] P. C. Abreu, M. Bayat, A. M. Pascoal, J. Botelho, P. GŮis, J. Ribeiro, M. Ribeiro, M. Rufino, L. SebastiŃo, and H. Silva, "Formation control in the scope of the morph project. part ii: Implementation and results," in 4th IFAC Workshop onNavigation, Guidance and Controlof Underwater VehiclesNGCUV 2015, vol. 48, no. 2. Girona, Spain: IFAC-PapersOnLine, 28-30 April 2015 2015, pp. 250-255. [Online]. Available: http://dx.doi.org/10.1016/j.ifacol.2015.06.041

[14] P. C. Abreu, M. Bayat, J. Botelho, P. Gois, J. Gomes, A. Pascoal, J. Ribeiro, M. Ribeiro, M. Rufino, L. Sebastiao, and H. Silva, "Cooperative formation control in the scope of the ec morph project: Theory and experiments," in Proceedings of MTS/IEEE Oceans'15 - Genova, May 2015, pp. 1-7. [Online]. Available: http://dx.doi.org/10.1109/OCEANS-Genova.2015.7271697

[15] D. De Palma, G. Indiveri, and A. M. Pascoal, "A null-space-based behavioral approach to single range underwater positioning," in 10th IFAC Conference on Manoeuvring and Control of Marine Craft MCMC 2015, vol. 48, no. 16, Copenhagen, 24-26 August 2015, pp. 55-60. [Online]. Available: http://dx.doi.org/10.1016/j.ifacol.2015.10.258

[16] A. S. Klyubin, D. Polani, and C. L. Nehaniv, "Empowerment: a universal agent-centric measure of control," in Prooceding of IEEE Congress on Evolutionary Computation, 2005., vol. 1, Sept 2005, pp. 128-135. [Online]. Available: http://dx.doi.org/10.1109/CEC.2005.1554676

[17] - "Keep your options open: An information-based driving principle for sensorimotor systems," PLOS ONE, vol. 3, no. 12, p. e4018, Dec 2008. [Online]. Available: http://dx.doi.org/10.1371/journal.pone.0004018

[18] T. Jung, D. Polani, and P. Stone, "Empowerment for continuous agentenvironment systems," Adaptive Behavior, vol. 19, no. 1, pp. 16-39, 2011. [Online]. Available: http://dx.doi.org/10.1177/1059712310392389

[19] T. Furfaro and J. Alves, "An application of distributed long baseline - node ranging in an underwater network," in Underwater Communications and Networking (UComms), 2014, Sestri Levante, Italy, Sept 2014, pp. 1-5. [Online]. Available: http://dx.doi.org/10.1109/UComms.2014.7017126

[20] V. Ludovico, J. Gomes, J. Alves, and T. C. Furfaro, "Joint localization of underwater vehicle formations based on range difference measurements," in Underwater Communications and Networking (UComms), 2014, Sestri Levante, Italy, Sept 2014, pp. 1-5. [Online]. Available: http://dx.doi.org/10.1109/UComms.2014.7017127

[21] A. Vermeij and A. Munafo, "A robust, opportunistic clock synchronization algorithm for ad hoc underwater acoustic networks," IEEE Journal of Oceanic Engineering, vol. 40, no. 4, pp. 841-852, Oct 2015. [Online]. Available: http://dx.doi.org/10.1109/JOE.2015.2469955 